\title{
New approach for tanh and extended-tanh methods with applications on Hirota-Satsuma equations
}

\author{
HASSAN A. ZEDAN \\ Mathematics Department, Faculty of Education, Kafr El-Sheikh University \\ Kafr El-Sheikh, Egypt \\ E-mail: hassanzedan2003@yahoo.com
}

\begin{abstract}
In this work, we establish new exact solutions for the Hirota-Satsuma equations. New approach for the tanh is used and extended tanh methods to construct traveling wave solutions in terms of a hyperbolic tangent functions. New families of solitary wave solutions and periodic solutions are also obtained for Hirota-Satsuma equations. Our approach is reduce the size of the computational adopted in other techniques without any conditions to apply on any system of partial differential equations.
\end{abstract}

Mathematical subject classification: 35K99, 35P05, 35P99.

Key words: Tanh method, extended tanh method, Hirota-Satsuma equations, traveling wave solutions, solitary wave solutions.

\section{Introduction}

The world around us is inherently nonlinear. The Hirota-Satsuma equations are widely used as models to describe complex physical phenomena in various fields of science, especially in fluid mechanics, solid stat physics, plasma physics. Various methods have been used to explore different kinds of solutions of physical models described by nonlinear PDEs. One of the basic physical problems for those models is obtaining their traveling wave solutions. Concepts like solitons, peakons, kinks, breathers, cusps and compacton are now being thoroughly investigated in the scientific literature [1-3]. During the past decades, quite a 
few methods for obtaining explicit traveling and solitary wave solutions of the Hirota-Satsuma equations have led to a variety of powerfull methods, such as inverse scattering method [4], bilinear transformation [5], the tanh-sech method [6], Backlund and Darboux transform [7], Hirota [8], tanh-function method [910]. Moreover these methods, extended tanh-function method [11-12], modified extended tanh-function method [13-14] and homogeneous balance method [15].

The extended tanh-function method and the modified extended tanh-function method belong to a class of methods called sub-equation method for which these appears few basic relationships among the complicated NLPDEs in the study and some simple and solvable nonlinear ordinary equations.

In this work, we introduce a matrix spectral problem with three potentials and propose a corresponding hierarchy of nonlinear evaluation equations. An interesting equation in the hierarchy is a generalization of the Hirota-Satsuma coupled kdv equations:

$$
\begin{aligned}
u_{t} & =\frac{1}{2} u_{x x x}-3 u u_{x}+3(v w)_{x}, \\
v_{t} & =-v_{x x x}+3 u v_{x}, \\
w_{t} & =-w_{x x x}+3 u w_{x} .
\end{aligned}
$$

\section{Tanh method}

In this section, we will try to search for a new analytical solutions for system (1)-(3), by using tanh method [12, 16]. This technique has been proven to be very powerful in finding travelling-wave solutions. We represent the tanh method for the problems (1)-(3) which is a workable and universal solution method that can be used to find exact as well as approximate solutions. This technique is based on the fact that in many cases traveling-wave solutions can be written in terms of a hyperbolic tangents.

Let the boundary conditions be

$$
\begin{aligned}
& u(x, t) \rightarrow 0, \quad v(x, t) \rightarrow 0 ; \quad x \rightarrow-\infty \\
& v(x, t) \rightarrow v_{\infty}, \quad w(x, t) \rightarrow 0 ; \quad x \rightarrow \infty
\end{aligned}
$$

To find possible travelling-wave solution, first we introduce the independent variable $\xi=c(x-v t)$, then, substituting $\xi=c(x-v t)$ into Eqs. (1)-(3), 
we get:

$$
\begin{gathered}
c^{2} \frac{d^{3} U(\xi)}{d \xi^{3}}+6 V(\xi) \frac{d W(\xi)}{d \xi}+6 W(\xi) \frac{d V(\xi)}{d \xi} \\
-6 U(\xi) \frac{d U(\xi)}{d \xi}+2 v \frac{d U(\xi)}{d \xi}=0, \\
c^{2} \frac{d^{3} V(\xi)}{d \xi^{3}}-3 U(\xi) \frac{d V(\xi)}{d \xi}-v \frac{d V(\xi)}{d \xi}=0, \\
c^{2} \frac{d^{3} W(\xi)}{d \xi^{3}}-3 U(\xi) \frac{d W(\xi)}{d \xi}-v \frac{d W(\xi)}{d \xi}=0,
\end{gathered}
$$

where the constants ( $c$ and $v$ ) represent (positive) wave number and (positive) velocity of the travelling-wave, $u(x, t)=U(\xi), v(x, t)=V(\xi)$ and $w(x, t)=W(\xi)$.

Secondly, substituting $Y=\tanh \xi$ into Eqs. (6)-(8) gives:

$$
\begin{aligned}
c^{2} & {\left[\left(1-Y^{2}\right)^{2} \frac{d^{3} U(Y)}{d Y^{3}}-6 Y\left(1-Y^{2}\right) \frac{d^{2} U(Y)}{d Y^{2}}-2\left(1-3 Y^{2}\right) \frac{d U(Y)}{d Y}\right] } \\
& +6 V(Y) \frac{d W(Y)}{d Y}+6 W(Y) \frac{d V(Y)}{d Y}-6 U(Y) \frac{d U(Y)}{d Y} \\
& +2 v \frac{d U(Y)}{d Y}=0, \\
c^{2} & {\left[\left(1-Y^{2}\right)^{2} \frac{d^{3} V(Y)}{d Y^{3}}-6 Y\left(1-Y^{2}\right) \frac{d^{2} V(Y)}{d Y^{2}}-2\left(1-3 Y^{2}\right) \frac{d V(Y)}{d Y}\right] } \\
& -3 U(Y) \frac{d V(Y)}{d Y}-v \frac{d V(Y)}{d Y}=0, \\
c^{2} & {\left[\left(1-Y^{2}\right)^{2} \frac{d^{3} W(Y)}{d Y^{3}}-6 Y\left(1-Y^{2}\right) \frac{d^{2} W(Y)}{d Y^{2}}-2\left(1-3 Y^{2}\right) \frac{d W(Y)}{d Y}\right] } \\
& -3 U(Y) \frac{d W(Y)}{d Y}-v \frac{d W(Y)}{d Y}=0 .
\end{aligned}
$$

where $U(\xi) \rightarrow U(Y), V(\xi) \rightarrow V(Y)$ and $W(\xi) \rightarrow W(Y)$.

Consequently, the boundary conditions reduce to the conditions with respect to the variable with respect to the variable $Y$ :

$$
\begin{array}{lll}
U(Y) \rightarrow 0, & V(Y) \rightarrow 0 ; & Y \rightarrow-1 \\
V(Y) \rightarrow V_{\infty}, & W(Y) \rightarrow 0 ; & Y \rightarrow 1
\end{array}
$$


To find an exact solution,we consider a finite expansion of $Y$ in the following form:

$$
U(Y)=\sum_{m=0}^{M} a_{m} Y^{m}, \quad V(Y)=\sum_{n=0}^{N} b_{n} Y^{n}, \quad W(Y)=\sum_{h=0}^{H} c_{h} Y^{h} .
$$

Substitution of Eq. (14) into Eqs. (9)-(11) and balancing the highest order derivative terms with the nonlinear terms, gives

$$
M=N=H=2 .
$$

Taking account of Eq. (15) with Eq. (14), we obtain

$$
\begin{gathered}
U(Y)=a_{0}+a_{1} Y+a_{2} Y^{2}, \\
V(Y)=b_{0}+b_{1} Y+b_{2} Y^{2}, \\
W(Y)=c_{0}+c_{1} Y+c_{2} Y^{2} .
\end{gathered}
$$

By using Eqs. (12) and (13) into Eqs. (16)-(18), we get:

$$
\begin{aligned}
U(Y \rightarrow-1) & =a_{0}-a_{1}+a_{2}=0 \\
a_{1} & =a_{0}+a_{2} \\
U(Y) & =a_{0}+\left(a_{0}+a_{2}\right) Y+a_{2} Y^{2}=(1+Y)\left(a_{0}+a_{2} Y\right) \\
U(Y) & =A_{1}(1+Y)\left(1+B_{1} Y\right) ; \quad A_{1}=a_{0}, B_{1}=\frac{a_{2}}{a_{1}} \\
V(Y \rightarrow-1) & =b_{0}-b_{1}+b_{2}=0 \\
b_{1} & =b_{0}+b_{2} \\
V(Y) & =b_{0}+\left(b_{0}+b_{2}\right) Y+b_{2} y^{2}=(1+Y)\left(b_{0}+b_{2} Y\right) \\
V(Y) & =A_{2}(1+Y)\left(1+B_{2} Y\right) ; \quad A_{2}=b_{0}, B_{2}=\frac{b_{2}}{b_{1}} \\
W(Y \rightarrow 1) & =c_{0}+c_{1}+c_{2}=0 \\
c_{1} & =-c_{0}-c_{2} \\
W(Y) & =c_{0}-\left(c_{0}+c_{2}\right) Y+c_{2} y^{2}=(1-Y)\left(c_{0}-c_{2} Y\right) \\
W(Y) & =A_{3}(1-Y)\left(1-B_{3} Y\right) ; \quad A_{3}=c_{0}, B_{3}=\frac{c_{2}}{c_{1}},
\end{aligned}
$$

where $A_{1}, B_{1}, A_{2}, B_{2}, A_{3}$ and $B_{3}$ are constants and can be determined. 
After substitution of Eqs. (19)-(21) into Eqs. (6)-(8), and eliminating terms having equal power in $Y$, we get the following:

$$
\begin{aligned}
& 3 A_{1}^{2}+3 A_{1}^{2} B_{1}-3 A_{2} A_{3} B_{2}+3 A_{2} A_{3} B_{3}+A_{1} c^{2}+A_{1} B_{1} c^{2} \\
& \quad+A_{1} v-A_{1} B_{1} v=0 \\
& 3 A_{1}^{2}+6 A_{2} A_{3}+12 A_{1}^{2} B_{1}+3 A_{1}^{2} B_{1}^{2}+6 A_{2} A_{3} B_{2} B_{3} \\
& \quad+8 A_{1} B_{1} c^{2}-2 A_{1} B_{1} v=0 \\
& 3 A_{1}^{2} B_{1}+3 A_{1}^{2} B_{1}^{2}+3 A_{2} A_{3} B_{2}-3 A_{2} A_{3} B_{3}-A_{1} c^{2}-A_{1} B_{1} c^{2}=0 \\
& A_{1}^{2} B_{1}^{2}-2 A_{2} A_{3} B_{2} B_{3}-2 A_{1} B_{1} c^{2}=0 \\
& -3 A_{1} A_{2}-3 A_{1} A_{2} B_{2}-2 A_{2} c^{2}-2 A_{2} B_{2} c^{2}-A_{2} v-A_{2} B=0 \\
& -3 A_{1} A_{2}-3 A_{1} A_{2} B_{1}-9 A_{1} A_{2} B_{2}-3 A_{1} A_{2} B_{1} B_{2} \\
& \quad-16 A_{2} B_{2} c^{2}-2 A_{2} B_{2} v=0 \\
& -3 A_{1} A_{2} B_{1}-6 A_{1} A_{2} B_{2}-9 A_{1} A_{2} B_{1} B_{2}+6 A_{2} c^{2}+6 A_{2} B_{2} c^{2}=0 \\
& -6 A_{1} A_{2} B_{1} B_{2}+24 A_{2} B_{2} c^{2}=0 \\
& 3 A_{1} A_{3}+3 A_{1} A_{3} B_{3}+2 A_{3} c^{2}+2 A_{3} B_{3} c^{2}+A_{3} v+A_{3} B_{3} v=0 \\
& 3 A_{1} A_{3}+3 A_{1} A_{3} B_{1}-3 A_{1} A_{3} B_{3}+3 A_{1} A_{3} B_{1} B_{3} \\
& \quad-16 A_{3} B_{3} c^{2}-2 A_{3} B_{3} v=0 \\
& 3 A_{1} A_{3} B_{1}-6 A_{1} A_{3} B_{3}-3 A_{1} A_{3} B_{1} B_{3}-6 A_{3} c^{2}-6 A_{3} B_{3} c^{2}=0 \\
& -6 A_{1} A_{3} B_{1} B_{3}+24 A_{3} B_{3} c^{2}=0
\end{aligned}
$$

In order to obtain $A_{1}, B_{1}, A_{2}, B_{2}, A_{3}, B_{3}$, we can solve Eqs. (22)-(33). Consequently we get

$$
A_{1}=-2 c^{2}, \quad B_{1}=-1, \quad A_{2}=\frac{4 c^{4}}{A_{3}}, \quad B_{2}=0, \quad B_{3}=0, \quad v=4 c^{2} .
$$

Substituting the above solution into Eqs. (19)-(21), we have the following analytical solutions:

$$
\begin{aligned}
& U(Y)=2 c^{2}(1-Y)(1+Y), \\
& V(Y)=\frac{4 c^{4}}{A_{3}}(1+Y), \\
& W(Y)=A_{3}(1-Y) ; \quad v=4 c^{2} \quad \text { with } \quad Y=\tanh [c(x-v t)] .
\end{aligned}
$$

where $A_{3}=2 c^{2}$. 


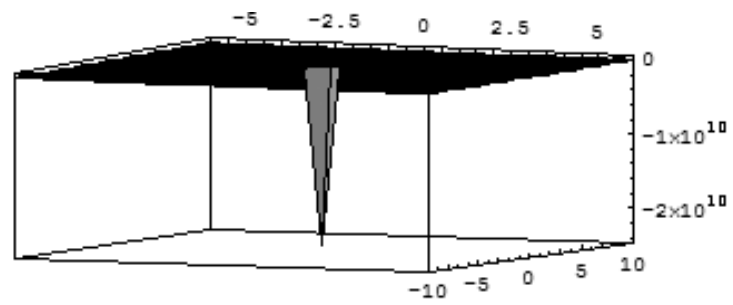

Figure 1 - The graph shows the solution for $u(x, t)$.

To find the wave number $(\mathbf{c})$, we use the boundary condition

$$
\begin{aligned}
V(Y \rightarrow 1) & =V_{\infty}, \\
\Longrightarrow c^{2} & =\frac{1}{4} V_{\infty}, \\
\Longrightarrow v & =V_{\infty} .
\end{aligned}
$$

It is well known that (wave-number) $c=109737.3$

$$
V_{\infty}=4.816910004516 \times 10^{10}, \quad v=4.816910004516 \times 10^{10}
$$

Then, Eqs.(1)-(3) have the following analytical solutions:

$$
\begin{aligned}
u(x, t) & =\frac{-1}{2} V_{\infty}[1-\tanh [c(x-v t)]][1+\tanh [c(x-v t)]], \\
v(x, t) & =\frac{1}{2} V_{\infty}[1+\tanh [c(x-v t)]], \\
w(x, t) & =\frac{1}{2} V_{\infty}[1-\tanh [c(x-v t)]],
\end{aligned}
$$

with $c, v, V_{\infty}$, are given.

\section{Extended tanh method}

In this section, we solve the Hirota-Satsuma coupled KdV equations by extended tanh method. This method is used to derive explicit travelling-wave solutions. New families of solitary wave solutions and periodic solutions are formally obtained for these equations. The method is used to reduce the computational size compared to the other techniques [16-20] without any conditions to apply on any system of partial differential equations. 


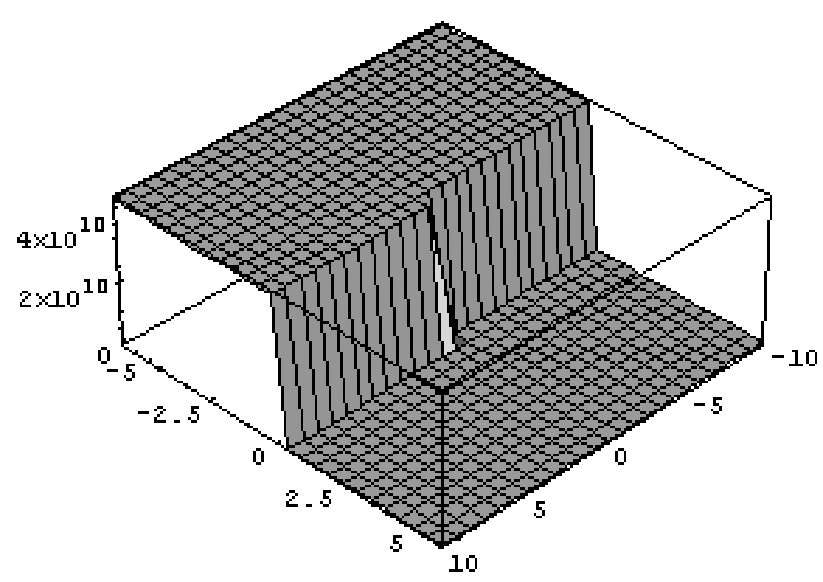

Figure 2 - The graph shows the solution for $v(x, t)$.

Using the wave variable $\xi=x-c t$, Eqs. (1)-(3) reduce to ODEs in the following form:

$$
\begin{aligned}
-c U^{\prime} & =\frac{1}{2} U^{\prime \prime \prime}-3 U U^{\prime}+3(V W)^{\prime}, \\
-c V^{\prime} & =-V^{\prime \prime \prime}+3 U V^{\prime}, \\
-c W^{\prime} & =-W^{\prime \prime \prime}+3 U W^{\prime},
\end{aligned}
$$

where $u(x, t) \rightarrow U(\xi), v(x, t) \rightarrow V(\xi)$ and $w(x, t) \rightarrow W(\xi)$.

Integrating Eq. (41) with respect to $(\xi)$, we get:

$$
-c U=\frac{1}{2} U^{\prime \prime}-\frac{3}{2} U^{2}+3(V W)
$$

where the constant of integration is equal to zero.

The solution of the reduced equations can be expressed as a finite power series in $Y$ in the form:

$$
\begin{aligned}
& u(x, t)=S_{1}(Y)=\sum_{m=0}^{M} a_{m} Y^{m}, \\
& v(x, t)=S_{2}(Y)=\sum_{n=0}^{N} b_{n} Y^{n}, \\
& w(x, t)=S_{3}(Y)=\sum_{h=0}^{H} c_{h} Y^{h},
\end{aligned}
$$




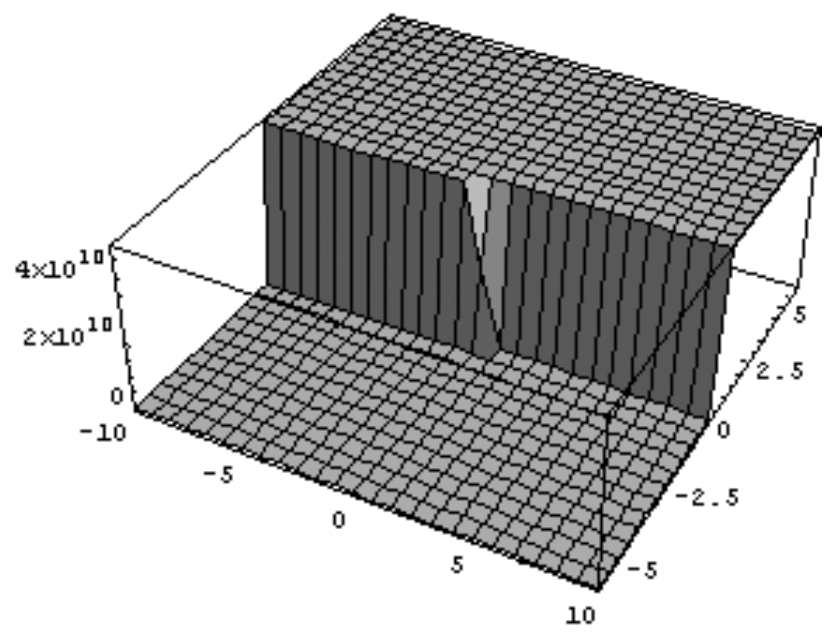

Figure 3 - The graph shows the solution for $w(x, t)$.

where $M, N$ and $H$ can be determined. By balancing between the highest derivative with the nonlinear terms, in Eqs. (42)-(44) gives

$$
M=N=H=2
$$

Substituting Eq. (46) into Eqs. (45), we find that:

$$
\begin{gathered}
u(x, t)=S_{1}(y)=a_{0}+a_{1} Y+a_{2} Y^{2}, \\
v(x, t)=S_{2}(y)=b_{0}+b_{1} Y+b_{2} Y^{2}, \\
w(x, t)=S_{3}(y)=c_{0}+c_{1} Y+c_{2} Y^{2} .
\end{gathered}
$$

Substituting $Y=\tanh (\mu \xi)$ in Eqs. (42)-(44), with the aid of Eqs. (47)-(49), and equating the coefficients of each power of $Y$ to zero, we obtain a system of algebraic equations of the parameters $a_{0}, a_{1}, a_{2}, b_{0}, b_{1}, b_{2}, c_{0}, c_{1}, c_{2}$, namely:

$$
\begin{aligned}
& -3 a_{0}^{2}+2 a_{0} c+6 b_{0} c_{0}+2 a_{2} \mu^{2}=0, \\
& -6 a_{0} a_{1}+2 a_{1} c+6 b_{1} c_{0}+6 b_{0} c_{1}-2 a_{1} \mu^{2}=0, \\
& -3 a_{1}^{2}-6 a_{0} a_{2}+2 a_{2} c+6 b_{2} c_{0}+6 b_{1} c_{1}+6 b_{0} c_{2}-8 a_{2} \mu^{2}=0, \\
& -6 a_{1} a_{2}+6 b_{2} c_{1}+6 b_{1} c_{2}+2 a_{1} \mu^{2}=0, \\
& -3 a_{2}^{2}+6 b_{2} c_{2}+6 a_{2} \mu^{2}=0, \\
& -3 a_{0} b_{1}-b_{1} c-2 b_{1} \mu^{2}=0,
\end{aligned}
$$




$$
\begin{aligned}
& -3 a_{1} b_{1}-6 a_{0} b_{2}-2 b_{2} c-16 b_{2} \mu^{2}=0, \\
& -3 a_{2} b_{1}-6 a_{1} b_{2}+6 b_{1} \mu^{2}=0 \\
& -6 a_{2} b_{2}+24 b_{2} \mu^{2}=0 \\
& -3 a_{0} c_{1}-c c_{1}-2 c_{1} \mu^{2}=0 \\
& -3 a_{1} c_{1}-6 a_{0} c_{2}-2 c c_{2}-16 c_{2} \mu^{2}=0 \\
& -3 a_{2} c_{1}-6 a_{1} c_{2}+6 c_{1} \mu^{2}=0 \\
& -6 a_{2} c_{2}+24 c_{2} \mu^{2}=0
\end{aligned}
$$

Using symbolic software Mathematica to solve the algebraic equations (50)-(62), we obtain the following set of distinct solutions of parameters. The set is given the following cases:

\section{Case 1:}

$$
\begin{aligned}
& a_{1}=c_{1}=b_{1}=0 \\
& a_{2}=4 \mu^{2}, \quad b_{2}=\frac{4 \mu^{4}}{c_{2}} \\
& b_{0}=\frac{1}{3}\left(\frac{A \pm B}{c_{2}}\right), \quad c_{0}=\frac{1}{12 \mu^{4}}(A \pm B) c_{2} \\
& c=-3 a_{0}-8 \mu^{2}
\end{aligned}
$$

where

$$
A=12 a_{0} \mu^{2}+24 \mu^{4}, \quad B=\sqrt{6} \mu^{2} \sqrt{\left(15 a_{0}^{2}+80 a_{0} \mu^{2}+104 \mu^{4}\right)}
$$

and $a_{0}, c_{2}$ are arbitrary constants. In this case, the soliton solutions take the form:

$$
\begin{aligned}
& u(x, t)=a_{0}+4 \mu^{2} \tanh ^{2}\left[\mu\left(x+\left(3 a_{0}+8 \mu^{2}\right) t\right)\right], \\
& v(x, t)=\frac{1}{3}\left(\frac{A \pm B}{c_{2}}\right)+\frac{4 \mu^{4}}{c_{2}} \tanh ^{2}\left[\mu\left(x+\left(3 a_{0}+8 \mu^{2}\right) t\right)\right], \\
& w(x, t)=c_{2}\left(\frac{A \pm B}{12 \mu^{4}}+\tanh ^{2}\left[\mu\left(x+\left(3 a_{0}+8 \mu^{2}\right) t\right)\right]\right) .
\end{aligned}
$$

where $a_{0}, c_{2}$ are arbitrary constants. 
Figs. (4)-(6) represent the soliton solutions for Eq. (63), by taking different values for constants, for example $a_{0}=-1, \mu=0.5, c_{2}=-1$.

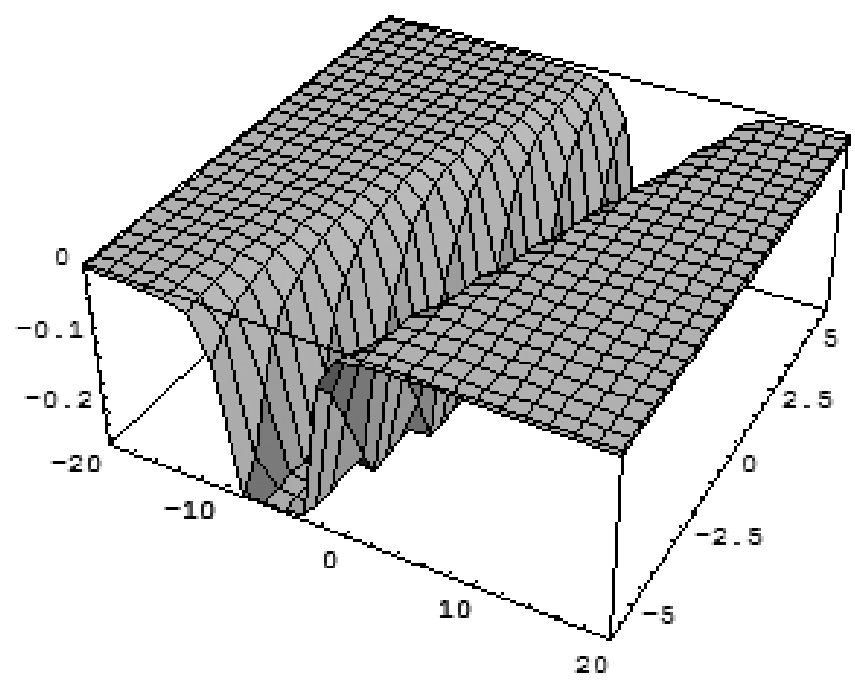

Figure 4 - The soliton solution for $u(x, t)$ in Case 1 when $a_{0}=-1, \mu=0.5, c_{2}=-1$.

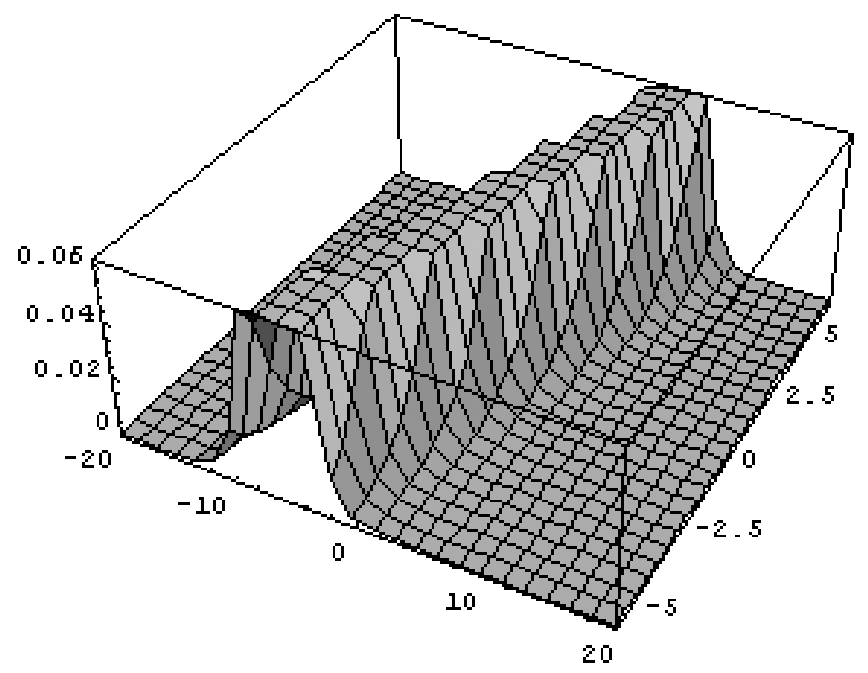

Figure 5 - The soliton solution for $v(x, t)$ in Case 1 when $a_{0}=-1, \mu=0.5, c_{2}=-1$. 


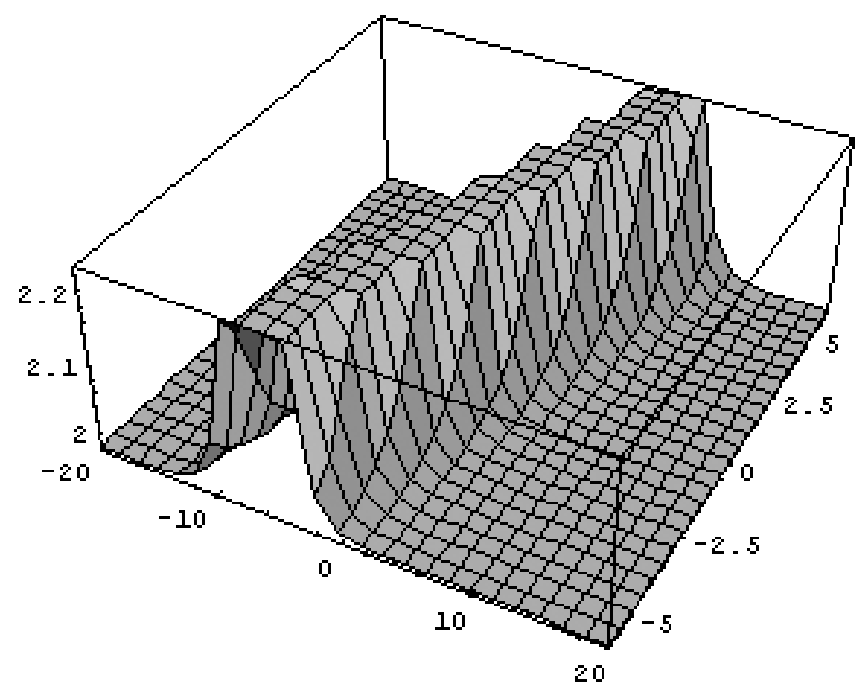

Figure 6 - The soliton solution for $w(x, t)$ in Case 1 when $a_{0}=-1, \mu=0.5, c_{2}=-1$.

\section{Case 2:}

$$
\begin{aligned}
& a_{1}=b_{2}=c_{2}=0, \\
& a_{2}=2 \mu^{2}, \\
& b_{0}= \pm\left[\frac{\left(a_{0} \mu+\mu^{3}\right)}{c_{1} B}\right], \quad b_{1}=\frac{4 \mu^{2}\left(a_{0}+\mu^{2}\right)}{c_{1}}, \\
& c_{0}=\mp \frac{c_{1} A}{\mu B}, \quad c=-3 a_{0}-2 \mu^{2},
\end{aligned}
$$

where

$$
A=\sqrt{-9 a_{0}^{2} \mu^{2}-4 a_{0} \mu^{2}+4 \mu^{4}}, \quad B=\sqrt{24 a_{0}+24 \mu^{2}}
$$

and $a_{0}, c_{1}$ are arbitrary constants. Thus, the soliton solutions take the form:

$$
\begin{aligned}
u(x, t) & =a_{0}+2 \mu^{2} \tanh ^{2}\left[\mu\left(x+\left(3 a_{0}+2 \mu^{2}\right) t\right)\right], \\
v(x, t) & =\frac{\left(a_{0}+\mu^{2}\right)}{c_{1}}\left[ \pm \frac{\mu}{B}+4 \mu^{2} \tanh \left[\mu\left(x+\left(3 a_{0}+2 \mu^{2}\right) t\right)\right]\right], \\
w(x, t) & =\mp \frac{c_{1} A}{\mu B}+c_{1} \tanh \left[\mu\left(x+\left(3 a_{0}+2 \mu^{2}\right) t\right)\right],
\end{aligned}
$$

where $a_{0}, c_{1}$ are arbitrary constants. 
Figs. (7)-(9) represent the soliton solutions for Eq. (64) as taking different values for constants, for example $a_{0}=-2.5, \mu=0.5, c_{1}=-1$.

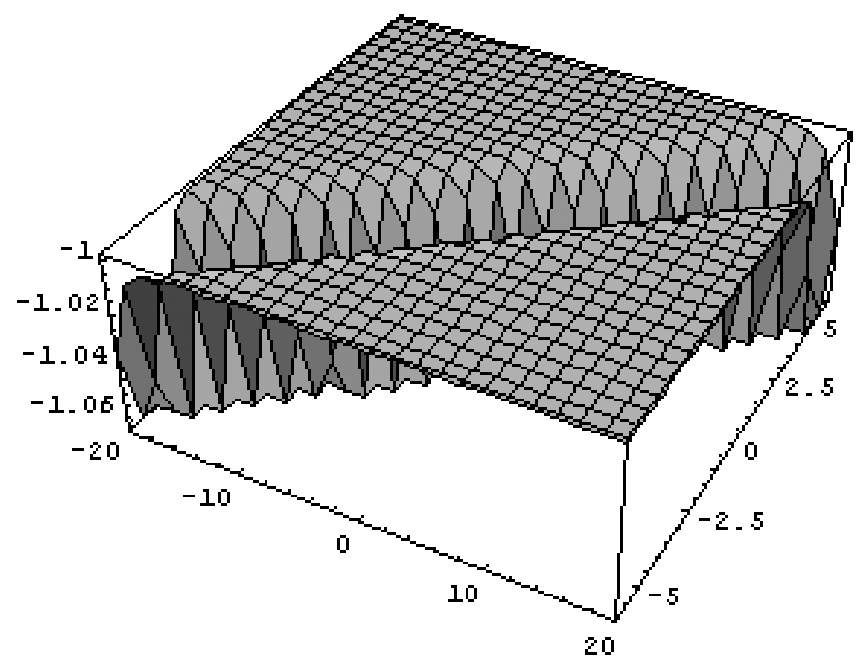

Figure 7-The soliton solution for $u(x, t)$ in Case 2 when $a_{0}=-1.5, \mu=0.5, c_{1}=-1$.

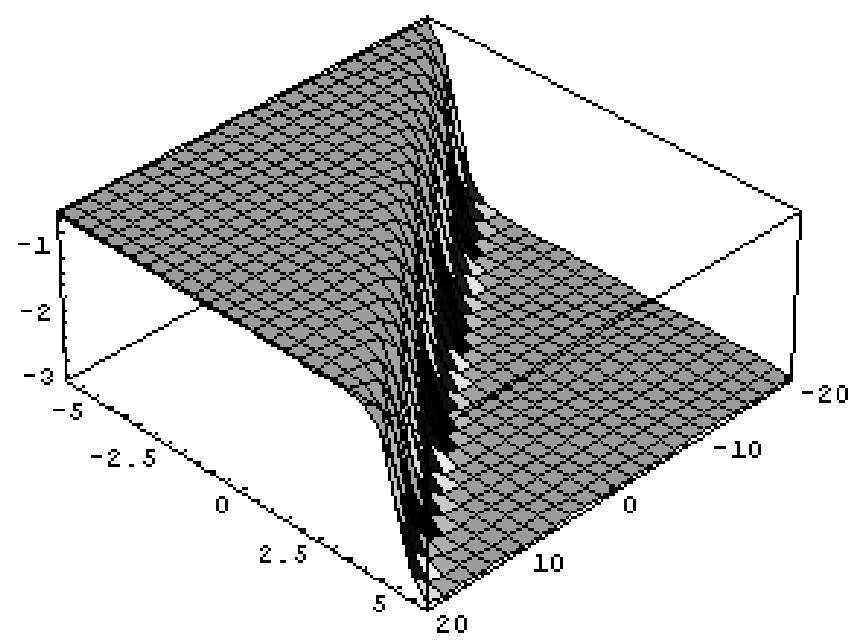

Figure 8 - The soliton solution for $v(x, t)$ in Case 2 when $a_{0}=-1.5, \mu=0.5, c_{1}=-1$. 


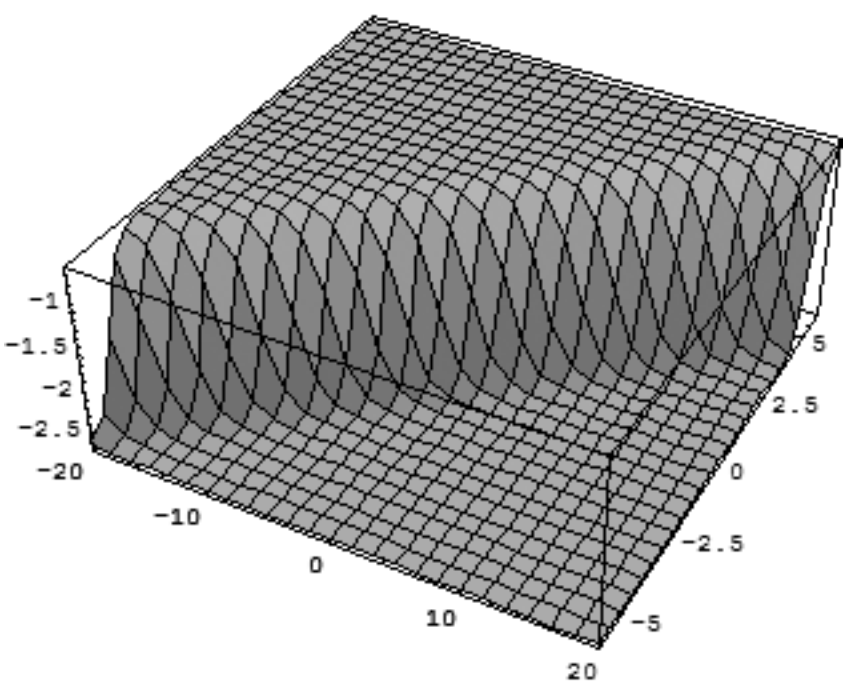

Figure 9-The soliton solution for $w(x, t)$ in Case 2 when $a_{0}=-1.5, \mu=0.5, c_{1}=-1$.

Acknowledgement. The author would like to thank the referee for his suggestions and comments on this article.

\section{REFERENCES}

[1] M. Wadati, Introduction to solitons. Pramana: J. Phys., 57(5-6) (2001), 841-7.

[2] M. Wadati, J. Phys. Soc. Jpn., 32 (1972), 1681.

[3] M. Wadati, J. Phys. Soc. Jpn., 34 (1973), 1289.

[4] M. Ablowitz and P.A. Clarkson, Soliton, Nonlinear Evolution Equations and Inverse Scattering. Cambridge Univ. Press, New York (1991).

[5] R. Hirota, Direct method of finding exact solutions of nonlinear evolution equations. In: R. Bullough and P. Caudrey (Editors). Backlund transformations. Berlin, Springer (1980). p. 1157-75.

[6] W. Malfliet and W.Hereman, Phys Sprica, 54 (1996), 569-75.

[7] M. Wadati, H. Sanuki and K. Konno, Prog. Theor. Phys., 53 (1975), 419.

[8] R. Hirota, Phys. Rev. Lett., 27 (1971), 1192.

[9] E.J. Parkes and B.R. Duffy, Comput. Phys. Commun., 98 (1996), 288.

[10] Z.B. Li and Y.P. Liu, Comput. Phys. Commun., 148 (2002), 526.

[11] E.G. Fan, Phys. Lett., A277 (2000), 212.

[12] B. Li, Y. Chen and H.Q. Zhang, Chaos, Soliton and Fractals, 15 (2003), 647. 
[13] E. Yomba, Chaos, Soliton and Fractals, 20 (2004), 1135.

[14] E. Yomba, Chaos, Soliton and Fractals, 22 (2004), 321.

[15] E. Fan and H. Zhang, Phys. Lett., A246 (1998), 403.

[16] A.M. Wazwaz, Chaos, Solitons and Fract., 28 (2006), 1005.

[17] A.M. Wazwaz, Comm. in Nonlinear Science and Numerical Simulation, 11 (2006), 376.

[18] A.M. Wazwaz, Math. and Comput. Modelling, 43 (2006), 802.

[19] A.M. Wazwaz, Chaos, Solitons and Fract., 28 (2006), 454.

[20] J. Weiss, M. Tabor and G. Carnevale, J. Math. Phys., 24 (1983), 522. 